\title{
Comparing Cefixime, Cefpodoxime and Ofloxacin as Anti-Microbial Agents and their Effects on Gut Microbiota
}

\author{
Kartik Singh ${ }^{1}$, Manju Mohan ${ }^{2}$, Shrishti Nautiyal ${ }^{3}$ \\ Guru Gobind Singh Indraprastha University, Delhi, India ${ }^{1}$ \\ University of Madras, Chennai, Tamil Nadu, India ${ }^{2}$ \\ Amity University, Noida, Uttar Pradesh, India ${ }^{3}$
}

\section{ABSTRACT}

\section{Article Info}

Volume 7, Issue 6

Page Number: 131-152

Publication Issue:

November-December-2020

Article History

Accepted: 10 Nov 2020

Published: 18 Nov 2020
The present review is based mainly on papers published between 190 and 2020 and gives information about the effects of Cefpodoxime, Ofloxacin and Cefixime on the gut flora, biological systems and its possible role in antibiotics. The main aim of this report is to highlight their role as an antibiotic and its relationship to the gut microbiota.

Keywords: Cefpodoxime, Ofloxacin, Cefixime, Gut Flora, Biological Systems, Biological Systems

\section{INTRODUCTION}

Effect of supplements with lactic acid bacteria and oligofructose on the intestinal microflora during administration of Cefpodoxime proxetil.

The human intestinal microflora is a complex ecosystem where microorganisms normally live in a stable relationship with their host. Antimicrobial agents can seriously disrupt the gastrointestinal microflora. Cefpodoxime proxetil is an orally administered cephalosporin which is used to treat upper and lower respiratory tract infections. Gastrointestinal symptoms, with increased gas production and diarrhea, are most common. Overgrowth of enterococci and yeasts and reduced numbers of enterobacteria are the most pronounced ecological effects in the intestinal microflora. To re- establish the balance of the intestinal microflora, Probiotics containing viable bacteria (especially bifidobacterial and lactobacilli) producing human lactic acid, have been used. The present investigation was performed to study the intestinal microflora, fecal $\mathrm{pH}$ and clinical status in healthy volunteers before, during and after administration of cefpodoxime proxetil, with or without supplements of B. longum, L. acidophilus and oligofructose.

Thirty healthy volunteers in three groups participated in a study of the effect on the intestinal microflora of oral supplementation with Bifidobacterium longum, Lactobacillus acidophilus and oligofructose, an indigestible oligosaccharide, during oral administration of cefpodoxime proxetil bd for 7 days. The mean age was 28 years ranging from 21-50 years. The subjects were made to visit the hospital before 
the investigation and on $21^{\text {st }}$ day of investigation. Those in group A also received an oral supplement with c.1011 cfu of B. longum BB 536 and L. acidophilus NCFB 1748 and 15 g oligofructose daily, those in group B received a placebo supplement with oligofructose only and those in group $\mathrm{C}$ received placebo without oligofructose, for 21 days. Stool specimens were collected before administration, on days 2, 4 and 7 of the antimicrobial administration periods and 2, 4, 7, 14 and 21 days after stopping the antimicrobial agent. The faecal samples were suspended in preproduced peptone-yeast extract medium, diluted, inoculated on non-selective or selective medium and processed as described by Heimdahl \& Nord. Aerobic and anaerobic microorganisms were identified using morphological, serological and biochemical tests and gas-liquid chromatography. The cytotoxin test was performed according to Aronsson et al. Strains of C. difficile, isolated from samples growing on selective C. difficile agar plates, were grown under anaerobic conditions. Strains of lactobacilli and bifidobacterial were collected and compared by PFGE to identify changes in the composition of strains of bifidobacterial in the volunteers during the study period and to differentiate the administered strains of $\mathrm{B}$. longum and L. acidophilus from endogenous bifidobacterial and lactobacilli. For aerobic microorganisms, no. of these organisms was decreased initially due to rapid disappearance of E. coli. The difference was significant only in group A and B. For anaerobic microorganisms, these were decreased mainly from the loss of bifidobacterial and also due to lactobacilli but was most pronounced in group A. Comparison of the collected strains of bifidobacterial with the administered strain of $B$. longum showed that one isolate from day 14 from one volunteer in group A was B. longum BB 536. In this volunteer the bifidobacterial had disappeared on day 4 but reappeared on day 14. The fluctuations of $\mathrm{pH}$ in faeces were most pronounced in group $\mathrm{A}$ and $\mathrm{B}$ during the investigation. The dry part of faecal mass significantly in all groups during administration of anti-microbial agents. Measurements correlated well with the subjective assessment of consistency of stools reported by the volunteers during the study. Concentration of triglycerides and cholesterol did not change throughout the study. Except one, all other volunteers completed the study without any side effects and it was observed that overall gastrointestinal symptoms were more in group A and $B$ than in group C. One subject in group A was excluded because of treatment with another antimicrobial agent. It has been reported that oligofructose and inulin promote the growth of bifidobacterial in vitro and in human supplementation. This effect was not observed in the present study, possibly because the effects of cefpodoxime proxetil on the intestinal microflora were so pronounced, that they may have swamped any effects of probiotic or carbohydrate administration on the endogenous bifidobacterial. This observation may be of clinical value but has to be investigated further in clinical studies, mainly of patients at risk of developing C.difficile disease.

This paper acknowledged the effects of various lactic acid bacteria and oligofructose on the intestinal microflora. There would be more research to be done, just to get a clear idea about the administration of cefpodoxime proxetil. The investigators did not totally satisfy the research with just giving less information about the $\mathrm{C}$. difficile and the diseases that are caused due to $\mathrm{C}$. difficile in the above mentioned 3 groups. Also, the researchers did not explain about the reappearance of the strains. This paper has many parts that could help in the research for further studies. More research can be done with the help of PFGE to identify the changes between various studies and on various functions of $\mathrm{C}$. difficile to cure premembranous otitis caused due to it, since 
the above-mentioned pathogenic activities of C. difficile.

Presence of Clostridium Difficile and Antibiotic and beta-lactamase activities in faeces of volunteers treated with oral cefixime, oral cefpodoxime proxetil, or placebo.

C.difficile is a supplement that causes inflammation of large intestine in patients given antibiotic treatment and is associated with production of enterotoxin (Toxin A) and cytotoxin (Toxin B). It is also a hospital acquired infection which has been isolated from infants as well as adults, but only few data are available of the strains that colonizes this subject. It is also associated with post antibiotic diarrhoea which is unclear psychopathological. Beta-lactamase is the main cause of post antibiotic diarrhoea, they minimize modifications of the intestinal flora. Various studies have also showed that Beta-lactamase promoted overgrowth of $\mathrm{C}$. difficile disease. Cefixime is an active compound and cefpodoxime proxetil is a prodrug without any antimicrobial activity until its ester bond is hydrolysed, these drugs only go partial intestinal absorption. There was no such information about cefpodoxime proxetil, but a close prodrug was found in the faeces. So, it was believed that even after the use of prodrug the active drug reached the colon and affected intestinal flora. After this, an investigation for C. difficile colonization was performed to see the difference after treatment with cefixime or cefpodoxime proxetil. An investigation for relationship of antibiotic and beta-lactamase activities with colonization and fecal excretion also performed. Genomic typing was used to differentiate between C. difficile isolates.

3 groups of 18 healthy adult volunteers (6 in each group) aged 24-42 years were assigned to a study of oral treatment with400mg of cefpodoxime, cefixime or placebo per day for 10 days. Fecal samples were obtained before treatment, daily during treatment and every other day for two weeks after treatment. C.difficile has Florissant cells colonies examined through microscope based on their shape position and API profile of colonies. Various isolates were tested for toxin B In a cytotoxicity assay with Chinese hamster ovary cells and toxin A by a commercially available immunoassay as recommended by manufacturer. Accuracy for both toxin A and toxin B were similar. Digested plugs were inserted into the wells of $1.5 \%$ agarose gel containing $0.5 \mathrm{X}$ TBE. This preparation was subjected to pulse field gel electrophoresis weather contour clamped homogeneous electric field device. Differences in proportions among groups were assessed by using Fisher's exact test.

The median number of specimens analyze from each volunteer was 16. C. difficile was not isolated from any of the 18 pre-treatment samples or from any samples from the six volunteers given placebo. It was present in the faeces of the three volunteers with loose stools. Two of these isolates were toxigenic in nature. In the present study, no statistical Association between the passage of loose stools and the shedding of toxigenic or non-toxigenic strains of $\mathrm{C}$ difficile was highlighted. Our data show no significant difference between the results of treatments with cefixime and with cefpodoxime proxetil, either in the proportion of volunteers excreting $C$ dificil or in the time that elapse before the excretion was detected.

C. Difficile Observed in the study was not significantly different from the four of six or the five of 10 previously reported for Volunteers treated with cefixime or cefpodoxime. C.difficile isolates showed that different toxigenic or non-toxigenic strains could be isolated on different days from the faeces of the same volunteer. C.difficile clones were isolated from the same volunteer on the same day, by this we 
conclude that either repeat colonization or co colonization by different strains.

Hydrolysis of antibiotic residues by fecal B-lactamases apparently afforded protection against different side effects. This research paper appeared to be of much use as it gave us proper information about different bacterial, antibiotic activities highlighted by cefixime, cefpodoxime and placebo. The researchers should have also focused more on the behaviour of cefixime and cefpodoxime proxetil with $\mathrm{C}$. difficile separately. As it is mentioned that we cannot conclude whether C. difficile clones show repeat colonization or cocolonization by different strains. The association of loose stools and toxigenic nature of C.defficil were not found. This paper provides a lot of content that can help with the research. More information about the toxic behaviour of the $\mathrm{C}$. difficile should be cleared. This paper also compares between the efficacy of cefixime and cefpodoxime in which cefpodoxime has a little less efficacy as compared to cefixime but rest it is of similar if not more efficacy.

\section{Cefpodoxime: pharmacokinetics and therapeutic uses}

Cefpodoxime is a semisynthetic $3^{\text {rd }}$ generation Cephalosporin which is available for use of prodrugs which is readily absorbed from the gut. It is effective against bacterial pathogens causing common infections. Its empirical formula is C22 H27 N 509 S2. Molecular weight $=557.6$, Active molecule of cefpodoxime covers a wide spectrum of bacteria and stable under beta lactamases. Pharmacokinetics which implies of time of absorption, metabolism, and excretion.

Tmax for absorption is 2 to 3 hours where $\mathrm{T} 1 / 2=2.09$ 2.84hrs.MIC90 varies from 7 to 12 hours after single oral dose. Urinary excretion is inversely proportional to dragon tail $90 \%$ of drug excreted as urine and $10 \%$ is faeces. The drug is a bacterial agent, after $D$ esterification by intestinal esterase drug inhibits their bacterial cell wall synthesis. Drug shows post antibiotic effect against gram positive but not against gram negative bacteria.

\section{THERAPEUTIC USES}

Cefpodoxime is twice as efficient as penicillium as it requires five days as compared to penicillium it required 10 days in treating upper respiratory tract infections it is used preferably due to its lower side effects and even compared with cefuroxime axetil and amoxy-clavolunate and have equal efficacy. Drug shows good efficacy when she used a step down the difficult patients who received parenteral cephalosporins initially. This drug has been found effective in treatment of patients with skin infections dealt for 7 to 14 days. Cure rates of people with UTI's with a single dose for three to 10 days of up to 95 to $97 \%$ whereas other reported cause was as high as $85 \%$.

\section{Review of clinical experience in the United States with cefpodoxime proxetil in adults with uncomplicated urinary tract infections}

Cefpodoxime proxetil is an orally administered prodrug possessing in vivo and in vitro bacterial activity comparable or better than other bacterial antibiotics. Results show that it has low mict against aerobic gram positive and gram-negative bacteria for example streptococci staphylococci etc. cefpodoxime has shown equal or better efficacy with other comparative antibiotics that is Cephalexin, cefaclor and ampicillin, it has shown better efficacy and safety with patients of UTI's. This paper was designed to examine the results of two separate and previously unpublished multicentre prospective studies that were: -

1. Comparative phase three clinical trials for efficacy.

2. Safety of patients with uncomplicated UTI's throughout US

Male and female patients aged 12 or above with bacteriuria or symptoms for UTI's could enrol. A preexamination was done for hypersensitivity in the past 
and asymptomatic patients were excluded of the study also patient undergoing other antibiotic medications are excluded. Eligible patients' urine was collected and culture, Patients are further allowed to go through trials one pathogen susceptible to go safe cefpodoxime and comparative agents was isolated in a quantity of $>=10^{\wedge} 5 \mathrm{CFU} / \mathrm{ml}$ of urine. Investigators scheduled patient for four protocol visits: -

1. Pre-treatment visit 48 hours before first dose.

2. Day 3-4 during treatment.

3. End of treatment (within 48 hours)

4. Post treatment visit.

Results were totally based on $2^{\text {nd }}$ and $4^{\text {th }}$ visit to patients according to the investigator.

A total of 568 patients with uncomplicated urinary tract infections are enrolled at 27 investigational sites.

378 patients were randomized to the cefpodoxime proxetil treatment group and 192 cefaclor group. A total of 206 patients out of which 132 treated with cefpodoxime proxetil and 74 treated with cefaclor Who are the chief boats bacteria logical and clinical key origin day 529 posttreatment visit returned at four to six weeks for long term follow up. results were similar for the two groups $80 \%$ of patients treated mid cefpodoxime proxetil and $82 \%$ treated with cefaclor showed no pathogen growth or signs and symptoms of UTI. 569 patients uncomplicated urinary tract infections are enrolled in a second comparative trial evaluating the efficacy and safety of amoxicillin. 384 patients were randomized to the safe food of Sam proxetil group and 185 to the amoxicillin group. The overall bacteriological cure rates were similar for the two treatment groups $81 \%$ for cefpodoxime proxetil and $70 \%$ for amoxicillin groups. Overall incidents of adverse experience were $31 \%$ cefpodoxime proxetil $35 \%$ cefaclor and $21 \%$ And mostly due to syphilis stories these were related to gastrointestinal infections, but no patients developed pseudomembranous colitis. Only one patient in Cefpodoxime proxetil group experienced an adverse event and was considered both serious and drug related. Vagina Candidiasis was most frequently reported genital tract problem and was found to be generally like all cephalosporinase but was highest in cefaclor. More patients with this adverse incident excluded and word treated with topical antifungal drug therapy afterwards.

This paper provided a severe data about the working of cefpodoxime proxetil with the patients that have uncomplicated UTIs. It indicated about the clinical cure rates of different antibacterial agents that were used in this research where, cefpodoxime proxetil was well tolerated. It is safe and efficient in the patients with uncomplicated UTIs and compared well with other antibacterial agents used under the research. This drug can be used in comparison to other cephalosporins as it is equally if not better in efficacy and can be compared against many other antibacterial agents because it is cost efficient, divided into 2 doses per day, has less recovery time and only $0.5 \%$ to $10 \%$ of side effects.

\section{Comparison of efficacy and safety of cefpodoxime and Amoxicillin clavulanate potassium in paediatric acute otitis media in children below two years: A prospective longitudinal study}

This research was based on Scientific understanding of AOM.Children with AOL acute otitis media are routinely treated with antimicrobial agents. Key factor to this research is the choice of antimicrobial drugs. Common bacteria and causing AOM in children Streptococcus pneumonia, Haemophilus influenzae etc. Amoxicillin-clavulanate potassium's high dose can treat middle ear at day 4-6 of treatment. It was recommended as a first line drug for AOM, By American Association of Paediatrics. They also recommended that cephalosporinase can be used as 
an alternate to treat PAOM. Date of 40 children that were clinically diagnosed with AOM aged between 6 months to 23 months was received over four months period. Children with hypersensitivity and below six months of age were excluded. Two groups were made:-

1. Amoxicillin dose for 10 days $10 \mathrm{mg} / \mathrm{kg} /$ day divided in 2 doses

2. Cefpodoxime dose for 10 days $10 \mathrm{mg} / \mathrm{kg} /$ day divided in 2 doses.

And the children showing side effects to these medications were excluded immediately. Efficacy parameter was number of children achieving treatment success in each group and it was based on AOM -SOS scores.

AOM-SOS score $<=1$ "clinically cured"

AOM-SOS score b/w 2-4 "clinical improvement"

Children were visited at the pre-treatment day, day 5 and day 10 .

The clinical success rates were $93.8 \%$ in Group A and $88.2 \%$ in Group B. P value 0.25 children out of the 16 analysing Group A achieved treatment success that is either clinical improvement or clinical cure, and the remaining 1 was characterized as treatment failure. In the Group B 15 children out of the 17 evaluated showed treatment success, that is either clinical improvement or clinical cure, and the remaining two were categorized as treatment failure. None of the children in Group B reported any $\mathrm{AE}$, these were mild AE's and did not require modification of drug therapy. Over all study profile of both the drugs was good.

This research paper compares the efficacy and safety of cefpodoxime against Amoxicillin- clavulanate potassium in infants having PAOM (aged 5-24 months).The American association of paediatrics suggested that other cephalosporins were used for an alternate in this treatment that's why, this research was designed to find whether cefpodoxime can be used as an alternative for PAOM. Further, the investigators found out that a 10-day cefpodoxime treatment for PAOM came in as a clinical success and was comparable to that of 10 days treatment of Amoxicillin- clavulanate potassium and can replace this treatment. This research proved that cefpodoxime proxetil is comparable against amoxicillin-clavulanate potassium treatment, it also means that this prodrug can be compared to similar drugs as Amoxicillin and can even replace several treatments with the same efficiency and safety indeed. Cefpodoxime can be used in both upper and lower respiratory tract infections, which further proves that it can replace many other treatment protocols as well.

\section{Cephalosporins associated with outer membrane vesicles released by Bacteroides spp. Protect gut pathogens and commensals against b-lactam antibiotics}

The adult human gastrointestinal (GI) tract accommodates a bacterial community (the microbiota) comprising trillions of cells that carry out vital functions for human health. This association involves co-evolved beneficial human - microbiota interactions that are altered as a result of many environmental factors. In particular, the presence of antibiotics can disturb colonic metabolism and absorption of vitamins, and can alter susceptibility to infection. The human GI tract microbiota is dominated by two bacterial phyla, Firmicutes and Bacteroidetes. Species of the genus Bacteroides, which constitute $30 \%$ of all bacteria in the human GI tract, are among the most resistant anaerobes to antibiotics, including penicillin and broadspectrum cephalosporins. Bacteroid species and strains were cultivated under anaerobic environment at $37^{\circ} \mathrm{C}$ in the brain heart infusion (BHI) medium complemented by $0.5 \mathrm{mg} / \mathrm{L}$ of haemin. Antibiotic 
tolerance markers in B. thetaiotaomicron were chosen using $1 \mathrm{mg} / \mathrm{L}$ of tetracycline, $5 \mathrm{mg} / \mathrm{L}$ of erythromycin or $200 \mathrm{mg} / \mathrm{L}$ of gentamicin. E. Coli strains GC10 converted with pGH014-based plasmids and HB101(pRK2013) were cultivated under agitation at $37{ }^{\circ} \mathrm{C}$ in the LB medium complemented by $100 \mathrm{mg} /$ $\mathrm{L}$ of spectinomycin and $50 \mathrm{mg} / \mathrm{L}$ of kanamycin, respectively. Electro-competent E. Coli cells were processed and transformed using the Sambrook and Russell methods (2001). Salmonella enterica serovar Typhimurium ATCC 14028 was grown at $37^{\circ} \mathrm{C}$ in the agitated LB medium.

The rate of degradation of cefotaxime following incubation with OMVs provided by different Bacteroid species was analysed using a disc susceptibility test. The tolerance of Salmonella Typhimurium and Bifidobacterium breve to cefotaxime in liquid culture in the presence of $\mathrm{B}$. thetaiotaomicron OMVs was assessed by calculation of growth of bacteria. The B.thetaiotaomicron BT 4507 gene encodes the beta-lactamase associated with CepA cephalosporinase of Bacteroides fragilis. The OMV created by B. Thetaiotaomicron and many other Bacteroid bacteria, besides Bacteroides ovatus, bore surface-associated beta-lactamases that could dissolve cefotaxime. Beta-Lactamase harbouring OMVs of B. thetaiotaomicron saved Salmonella Typhimurium and B. Breve from an otherwise fatal dose of cefotaxime. This research focuses on the discovery and dissemination of cephalosporinase genes in the human intestine species Bacteroides. This study demonstrates that, in addition to the secretion of bacterial cell periplasms, these cephalosporinases are also associated with OMVs developed by these bacteria; the findings are consistent with the role of these OMVs in degrading beta-lactam antibiotics remotely from their mother cell.
Whereas Gram-positive beta-lactamase-producing species largely release their enzymes into the surrounding environment, beta-lactamases of Gramnegative bacteria are typically assumed to be restricted to the periplasmic space of the cells, acting in accordance mostly with diffusion barrier of the outer membrane. In this study, the researchers demonstrated the that part of cephalosporinase BtCepA becomes aligned with membrane vesicles and this BtCepA - OMV interaction adequately diminishes substrate beta-lactam antibiotics present in the surrounding medium. The extracellular breakdown of beta-lactam antibiotics is only due to OMVs, since no release of the enzyme to the medium has been observed. It is well known that the use of antibiotics greatly influences the intestinal microbiota. Researchers concluded that Bacteroid species in human GI tract microbiota have the ability to shield Salmonella and potentially other pathogens and commensal microorganisms, such as probiotic B.breve by producing a significant number of cephalosporinase-coated OMVs. More broadly, the presence of cephalosporinase-coated OMVs in the colon could lead to the preservation of a healthy microbiota that protects against the adverse effects of antibiotic therapy.

This paper provides significant data about how cephalosporins associated with outer membrane vesicles released by Bacteroides spp. Protect gut pathogens and commensals against beta-lactam antibiotics. It shows that how bacteroid species in human gastrointestinal tract have ability to shield salmonella and potentially other pathogens as commensal microorganisms. Also, the presence of cephalosporinase- coated OMVs in colon leads to preservation of healthy gut microbiota. This paper provides study that shows cephalosporinases are also associated with OMVs developed by these bacteria; the findings are consistent with the role of these OMVs in degrading beta-lactam antibiotics remotely 
from their mother cell. How and where cephalosporinases work in gut microbiota.

\section{Impact of Cefpodoxime Proxetil and Amoxicillin on} the Normal Oral and Intestinal Microflora

Cefpodoxime is highly effective against a wide range of gram-positive and gram-negative bacteria except Enterococcus faecalis, Pseudomonas aeruginosa and Bacteroides fragilis. The aim of the present study was to study the oral and intestinal microflora before, during and after administration of cefpodoxime. proxetil and amoxicillin respectively, with regard to alterations in the ecological balance, concentrations of cefpodoxime and amoxicillin in saliva and faeces, and beta-lactamase production in the intestinal microflora. Twenty volunteers participated in a single-blind, randomized, comparative study of cefpodoxime proxetil versus amoxicillin. All participants were considered stable on the basis of their medical records and regular clinical laboratory examinations conducted before the start of the study and after the study. Ten volunteers, six females and four males (range 21-57 years), received $200 \mathrm{~g}$ cefpodoxime proxetil tablets every 12 for a week, and ten volunteers, five females and five males (range 2342 years), received $500 \mathrm{~g}$ amoxicillin tablets every 8 for a week. Both drugs were ingested with 50 litres of water and administered during meals.

The concentrations of cefpodoxime and amoxicillin in saliva and faeces were calculated by the agar well dilution method. The test medium was Antibiotic Medium No. 1 (Oxoid, UK). The indicator strain was Morganella morganii 313 VC3 for cefpodoxime and Sarcina lutea ATCC 9341 for amoxicillin. The specimens of saliva and stool were dissolved in the pre-reduced peptone-yeast extract medium, diluted, inoculated on selective media and examined. The sensitivity of species to cefpodoxime and amoxicillin was calculated by the calculation of minimum inhibitory concentrations (MICs). The MICs were evaluated using the agar dilution procedure. The beta-lactamase activity in faeces was determined spectrophotometrically with nitrocefin as Substrate.

Cefpodoxime and amoxicillin concentrations in saliva were very minimal. The lower concentration limit was $0.125 \mathrm{mg} / \mathrm{l}$ for both drugs. No measurable amounts of cefpodoxime or ampicillin were present in either of the samples of saliva. The amounts of cefpodoxime in faeces differed widely between the 10 subjects. No cefpodoxime could be observed in the faecal samples in seven volunteers, while high concentrations of cefpodoxime were identified in three volunteers. The mean concentrations of cefpodoxime in these three volunteers were $220 \mathrm{mg} /$ $\mathrm{kg}$ (range 7-630 g/ kg) on day 4; $430 \mathrm{mg} / \mathrm{kg}$ (range 290-700 mg / kg) on day 7; and $140 \mathrm{mg} / \mathrm{kg}$ (range 0.125-30 mg / kg) on day 9. Faecal samples obtained on days $0,2,11,14$ and 21 had no detectable cefpodoxime amounts. No measurable amoxicillin concentrations were found in any of the faecal samples from the amoxicillin community.

The administration of cefpodoxime proxetil produced only slight modifications to the oral microflora. The number of streptococci decreased although no major shifts were seen in other aerobic or anaerobic bacteria. In the gastrointestinal aerobic microflora, the number of streptococci, Escherichia coli and Klebsiella declined during cefpodoxime proxetil administration. There was a large overgrowth of enterococci during the administration period. Two subjects were colonised by high levels of staphylococci and yeast during the administration of cefpodoxime proxetil. The numbers of anaerobic cocci, lactobacilli, bifidobacterial and eubacteria were partially depressed, while the number of clostridia were greatly decreased. There are three subjects with the largest quantities of Clostridium difficile (> $5 \times 103$ $\mathrm{cf} \mu$ / g faeces) were the same subjects who seemed to 
have high amounts of cefpodoxime in their faecal samples.

The oral and intestinal microflora had returned to normal two weeks after the removal of cefpodoxime proxetil, with the exception of two volunteers still bearing Clostridium difficile. The administration of amoxicillin induced mild disruptions in aerobic oral microflora. Streptococci, micrococci, corynebacterial, bifidobacterial and Propionibacterium were partially depressed during amoxicillin administration, while no other aerobic or anaerobic bacterial groups were affected.

Aerobic intestinal microflora was partially affected by amoxicillin administration. There was a slight decrease in the number of streptococci and staphylococci. There was an overgrowth of Klebsiella in six volunteers and Enterobacter in two volunteers. There was no overgrowth of enterococci or yeast. In anaerobic intestinal microflora, amoxicillin administration has reduced only the number of eubacteria. Many other major anaerobic bacterial classes have remained unchanged. No colonisation with Clostridium difficile has been detected. Oral and intestinal microflora was shown to be normal two weeks after amoxicillin was ended. Amoxicillin treatment strongly induced the development of new colonising amoxicillin resistant microorganisms in normal faecal microflora. No serious side effects were recorded.

There was a correlation between cefpodoxime concentrations in the faeces and ecological disturbances in the intestinal microflora. Administration of amoxicillin also influenced alterations in the intestinal microflora, although to a slightly lesser extent than with cefpodoxime. Observation of no or very low amoxicillin levels in faeces is probable partly due to the high bioavailability of amoxicillin and partly because amoxicillin is hydrolysed more by beta-lactamases than by cefpodoxime.

Administration of both cefpodoxime proxetil and amoxicillin stimulates a high level of beta-lactamase activity in the faeces. Since cefpodoxime is more immune to beta-lactamases than to amoxicillin, it is more active in the intestinal tract and thus results in greater alteration of normal microflora.

This paper gives relevant survey about how these two antibiotics work and help in improvement of microbiota. It gives all answers like which one should be consider at what time. As it is randomized, and few side effects can be seen. Hence it does not provide clear evidence of their mechanics but surely give us few ideas about it. It also helps to easily compare between cefpodoxime and amoxicillin. This paper also shows that how they work on human gut microbiota.

\section{The effect of gastric $\mathrm{pH}$ and food on pharmacokinetics of new oral cephalosporins, cefpodoxime proxetil}

Sodium bicarbonate (1 gm) showed no effect on the pharmacokinetics of cefpodoxime proxetil. The pharmacokinetics of cefpodoxime proxetil and the effects of alteration of gastric $\mathrm{pH}$ under maximal acid stimulation with pentagastrin 6 and with nearcomplete acid neutralization or suppression with an H2-receptor antagonist (ranitidine), sodium bicarbonate, and aluminium.

The role of diet on the pharmacokinetic profile of cefpodoxime proxetil because food has been shown to increase the area under the plasma concentration time curve by $30 \%$ versus fasting conditions in Japanese men (Food produces many complex effects on gastrointestinal function, such as gastric acid secretion or gastric emptying, which might alter the pharmacokinetics of this antibiotic agent. For 
example, a delay in gastric emptying (induced by anticholinergic drugs) slightly increases the time to peak plasma concentration, but not the extent of absorption. Studies that describe the effects of alteration of one component of gastric physiology, gastric $\mathrm{pH}$, in conjunction with a series of wellcontrolled diets of varying composition, caloric density, and osmolality, could help to better understand the pharmacology of this cephalosporin antibiotic agent. Seventeen healthy, non-smoking adult males (aged 18 to 42 years) participated in the $\mathrm{pH}$ survey, while 16 participants (aged 18 to 41 years) participated in the food survey. All were within 20 percent of their ideal body weight $(73.7- \pm 12.0 \mathrm{~kg}$ in the $\mathrm{pH}$ study;71.5- $\pm 13.5 \mathrm{~kg}$ in the food study). Results of all renal, hepatic, haematological and urinary test, and stool tests for Clostridium difficile toxin is standard for all participants throughout each sample.

In the $\mathrm{pH}$ analysis ( $\mathrm{n}=17$ subjects), lead-in duration was conducted under fasting conditions, followed by random assignment to a four-way Penta gastrin crossover (6 $\mu \mathrm{g} / \mathrm{Kg}$, subcutaneously), ranitidine (150 mg orally, 10 and 2 hours before antibiotic dosing), sodium bicarbonate (12.6 g) or aluminium hydroxide $(120 \mathrm{cc})$. Gastric $\mathrm{pH}$ was measured by nasogastric aspirates before and 10 minutes after the treatment, right before the antibiotic was administered. In the food study ( $\mathrm{n}=16$ subjects), there were two periods of leading, one in fasting and one in normal diet, followed by randomization to a four-way crossover either of high or low protein diets or high or low-fat diets. There were six meals per diet. The antibiotic dose was taken at the mid span of the fourth meal.

Five-millilitre plasma samples were collected as an anticoagulant agent in EDTA freeze-dried tubes at specific times in relation to cefpodoxime proxetil dosing. Urine samples for the measurement of drug levels and creatinine clearance were pooled at 0 to 24 hours and 24 to 48 hours after dosing. After refrigerated centrifugation, separate plasma samples were frozen at-70 ${ }^{\circ} \mathrm{C}$ and stored for evaluation. Samples of urine were likewise frozen and stored.

Stool specimens for C. difficile toxin were done by two stage tissue culture assays. The first stage looked for cytopathic effects of the toxin on tissue cells. If such effects were detected, a specific antiserum against the toxin was added (the second stage of the assay) and the test was repeated. Gastric $\mathrm{pH}$ was measured in vitro with an electrode while Osmolality of diets was measured by freezing point depression. Peak plasma (C max) and area under plasma concentration time curve (AUC) were maximum during fasting and Penta gastrin periods and were 35 to 50 percent lower for all other periods ( $\mathrm{p}<0.0001$ ). Gastric $\mathrm{pH}$ and $\mathrm{C}$, and AUC were inversely related $(\mathrm{r}$ $=0.66$ and $\mathrm{r}=0.62, \mathrm{p}<0.0001$ for both). Effect of food; Cmax and AUC were 22 to 34 per cent higher for all diets than for the fasting cycle $(\mathrm{p}<0.0001)$, although the time for $\mathrm{Cmax}$ remained unchanged.

These studies have shown that absorption of cefpodoxime proxetil is better at low gastric $\mathrm{pH}$ or in the fed state, whereas the converse was appropriate for a number of agents that suppress stomach acidity (ranitidine, sodium bicarbonate, and aluminium hydroxide) which indicates that the role of gastrointestinal activity in the pharmacokinetic profile is complex. This paper provides us about the absorption of cefpodoxime under the effect of gastric $\mathrm{pH}$ and eating habits. This helps to know the ideal situation at which cefpodoxime works properly or ideally.

Cefpodoxime: Comparative Antibacterial Activity, Influence of Growth Conditions, and Bactericidal Activity 
Cefpodoxime proxetil is rapidly hydrolysed to cefpodoxime during absorption from the intestinal tract. The objective of this study was (a) to determine the influence of various growth conditions, i.e. inoculum size, $\mathrm{pH}$, and different media on the antibacterial activity of cefpodoxime, (b) to compare the in vitro activity of cefpodoxime, cefixime, cefotiam, cefuroxime and cefotaxime against a broad spectrum of freshly isolated bacterial strains.

Dependency of the temperature stability of cefpodoxime was determined using Bacillus subtilis as a test bacterium in Mueller-Hinton-broth at $\mathrm{pH} 7.4$ and in aqua distillate at $\mathrm{pH}$ 5.0. The effect of hydrogen concentration ( $\mathrm{pH} 6.2-7$ ) in MuellerHinton-broth and the influence of various inoculum sizes on antibacterial activity of cefpodoxime were tested using different gram-positive and gramnegative bacteria strains. The impacts of various media on the antibacterial activity of cefpodoxime against various pathogens were also tested, as was the influence of inactivated human serum added to the medium on cefpodoxime activity.

In addition, the minimal inhibitory concentration (MIC) of cefpodoxime was compared with the minimal bactericidal concentration (MBC). In the study of antibacterial activity of cefpodoxime as compared to other cephalosporins, Mueller-Hintonagar was used without or complemented by 5 per cent of ovine erythrocytes (Haemophilus influenzae strains). An inoculum of $1 \times 10^{\wedge} 5 \mathrm{CFU} / \mathrm{ml}$ was used and MICs were determined to be the lowest antibiotic concentration at which no visible growth was detected after $18 \mathrm{~h}$ at $36.5^{\circ} \mathrm{C}$. The MBC was identified as the lowest concentration resulting in a 99.9 percent reduction in CFU from the initial concentration of $1 \mathrm{x}$ $10^{\wedge} 5 \mathrm{CFU} / \mathrm{ml}$ inoculum at $18 \mathrm{~h}$ and $36.5^{\circ} \mathrm{C}$. Cefpodoxime was shown to be inhibitory at concentrations of $<1 \mathrm{mg}$ / L compared to 90 percent of the strains of Moraxella catarrhalis, Haemophilus influenzae, Escherichia coli (beta-Lactamase-negative strains), Klebsiella spp., Serratia spp., Proteus mirabilis, Proteus vulgarise, Providencia spp., and Salmonella spp. This antimicrobial activity of cefpodoxime was usually higher than that of cefuroxime and comparable to that of cefixime. Cefpodoxime was active at $<1 \mathrm{mg} / \mathrm{L}$ compared with $50 \%$ of the 13-Lactamase-producing members Escherichia coli, Enterobacter cloacae, Enterobacter aerogenes, Citrobacter spp. And Morganella morganii. Cefpodoxime has been shown to be strongly inhibitory against group A, B, and G streptococci and Streptococcus pneumoniae (MIC $90<0.015 \mathrm{mg} / \mathrm{l}$ ). The MICs of cefpodoxime and those of the other cephalosporins were $<2 \mathrm{mg} / 1$ for $>90$ percent of the strains of Staphylococcus aureus and Staphylococcus epidermidis, excluding cefixime which had no activity with MICs below $8 \mathrm{mg} / \mathrm{l}$ against these bacteria. Pseudomonas spp., Acinetobacter spp., Enterococcus spp. They were immune to cefpodoxime. The antibacterial activity of cefpodoxime was only to a minor degree influenced by different growth conditions except for high inoculum sizes against some beta- lactamase producing strains of gram-negative bacilli.

In view of the recorded in vitro antimicrobial activity of cefpodoxime, the prodrug cefpodoxime proxetil is expected to be very useful for the treatment of patients with respiratory and urinary tract infections following oral administration. This paper provides detailed study of cefpodoxime against various bacterial strains. It provides with detailed information of cefpodoxime and shows the working of it against various bacterial strain. The comparison between the functioning of cefpodoxime on different patients can be observed. 
Antibacterial activity of cefpodoxime proxetil in a pharmacokinetic in-vitro model

Cefpodoxime proxetil is a recent broad-spectrum oral cephalosporin. Ester, which is de-esterified to its active metabolite, cefpodoxime, in the intestine. It is active in vitro against Enterobacteriaceae, Staphylococcus aureus and Haemophilus influenzas at concentrations $<4 \mathrm{mg} / \mathrm{l}$. Cefpodoxime is also stable to beta-lactamases induced plasmids. The in-vitro sensitivity of bacteria to antibiotics expressed in MIC values does not generally represent the therapeutic efficacy of a medication, since these results are collected by continuous concentrations of drugs while in vivo the concentration of drugs varies drastically. The aim of this analysis was to determine the function of cefpodoxime under conditions that are similar to the in-vivo scenario. An in-vitro model is used to predict the serum concentration time curves of cefpodoxime proxetil collected from human volunteers.

All bacterial strains were clinical isolates except E. coli which was a laboratory strain K.12 W3110. The MIC values of the strains were evaluated by serial dilution in Mueller-Hinton broth in microlitre plates as per the guidelines of the NCCLS (1985). The invitro model simulates open one compartment pharmacokinetics by a dilution technique. Using constant volumes in the antibiotic and culture. In the culture vessel, flasks and a constant flow of antibioticfree broth from the reservoir can cause changes in drug concentrations according to the Bateman equation. For all bacteria nutrient broth $\mathrm{N} 1$ at a $\mathrm{pH}$ of 7-4 was used for precultures and during the experiments. Nutrient agar N1 was used to evaluate the feasible counts. For $\mathrm{H}$ influenzae all media were supplemented with 3 percent Fildes extract. Str. Pyogenes had been put on the blood agar.

The antibacterial activity of cefpodoxime proxetil was studied in an in vitro simulating dose regimen of 100 ,
200 and $400 \mathrm{mg}$. Strains from Klebsiella spp. Proteus mirabilis, Escherichia coli, Streptococcus pyogenes and Haemophilus influenzas were effectively reduced by $200 \mathrm{mg}$. Though for E.coli no dose-activity interaction was observed - the maximum effect was reached with a simulated dose of $100 \mathrm{mg}$ Staphylococcus aureus could be minimised efficiently only by a simulated dose of $400 \mathrm{mg}$. The lower doses showed lower activity stepwise. Apart from broad spectrum beta-lactamases such as SHV 2 or TEM 5 the presence of plasmid-coded beta-lactamases in E. Coli and $\mathrm{H}$. Influenza did not impair the antibacterial function of cefpodoxime proxetil.

The findings indicate that cefpodoxime was more competitive against Gram-negative bacteria than amoxycillin as compared to intramuscular cefotiam in the in-vitro form. It provides the ideal dosage of cefpodoxime and Antibacterial activity of cefpodoxime proxetil in a pharmacokinetic in-vitro model. This research helps to compare the amoxicillin with cefpodoxime. Strains from Klebsiella spp. Proteus mirabilis, Escherichia coli, Streptococcus pyogenes and Haemophilus influenzas were effectively reduced by $200 \mathrm{mg}$. Though for E.coli no dose-activity interaction was observed - the maximum effect was reached with a simulated dose of $100 \mathrm{mg}$ - Staphylococcus aureus could be minimised efficiently only by a simulated dose of $400 \mathrm{mg}$.

\section{Ofloxacin}

Ofloxacin and the gastrointestinal tract: a potential role in the treatment of bacterial enteritis

Ofloxacin is highly active against so many bacterial enteritis pathogens. Strong faecal amounts are rapidly reached after a single oral dosage and can continue for up to five days despite partial binding of the faeces. In addition, adequate levels of ofloxacin persist in pancreatic secretions and bile for 12 to $14 \mathrm{~h}$ following oral administration. Clinical results from multiple centres indicate a timely reaction when ofloxacin is 
prescribed once daily for shigellosis, sahnonellosis and other numerous enteric pathogens. These theoretical findings and clinical evidence indicate a need for once-daily oral treatment ofloxacin for bacterial diarrhoea.

\section{Oral flora:}

Edlund et al (1988) found only a small effect of ofloxacin, administered as a single oral dose of 400 $\mathrm{mg}$, on the oral microflora of patients undergoing gastric surgery. Branhamella strains were significantly reduced in numbers and returned to normal within a few weeks but the number of streptococci, micrococci and corynebacterial remained unchanged. Colonization with Gram-negative rods occurred. The anaerobic microflora was unaffected by ofloxacin except for suppression of Veillonella spp. between day 2 and day 7 in five of the 24 patients.

\section{Gastric flora:}

Edlund and at. (1988) analysed the influence of a single oral dose of $400 \mathrm{mg}$ Ofloxacin in the gastric microflora. Both anaerobic and aerobic bacteria have been killed in gastric secretions for 24 hours. No bacteria were found in six patients on day 0 , while five patients had $>10^{\wedge} 5 / \mathrm{ml}$. The number of bacteria was marginally greater when the $\mathrm{pH}$ was $>3-5$ than when the $\mathrm{pH}$ was $<3-5$ due either to a more conducive climate for bacterial replication or to more suitable conditions for drug action. In the study, colonisation of yeasts was seen in 13 patients, six of whom had $>10^{\wedge} 5 \mathrm{cfu} / \mathrm{ml}$ of gastric secretions. $>10$ $\wedge 5 \mathrm{cfu}$ yeasts $/ \mathrm{ml}$ gastric secretions were found in 15 of the 20 patients examined after 4 weeks.

\section{Faecal microflora}

Pecquet et al (1987) prescribed $400 \mathrm{mg}$ ofloxacin regularly to five normal volunteers for five days. The faecal concentration of Enterobacteriaceae decreased dramatically after administration and no strains were found in the faeces for four days after discontinuation of the treatment. Six days after withdrawing the drug, the amount of Enterobacteriaceae in faeces did not return to pre-treatment stages. The same trend was also found with enterococci, however a return to pretreatment thresholds was accomplished within four days of the completion of drug therapy. In addition, all volunteers have been colonised with Candida spp., though in limited numbers, after four days of observation. Anaerobic bacteria, on the other hand, remained at concentrations $>10^{\wedge} 10 \mathrm{cfu} / \mathrm{g}$ faeces. Emergency of tolerance to ofloxacin in the intestinal flora was not detected.

\section{Effect of antimicrobial therapy on the gastrointestinal} bacterial flora, infection and mortality in mice exposed to different doses of irradiation

The impact of antimicrobial therapy on intestinal flora, sepsis and mortality was examined in C3H/HEN irradiated female mice with 70,80 or $8-5 \mathrm{~Gy}$ or $60^{\wedge}$ Co. Metronidazole, penicillin, imipenem, gentamicin and ofloxacin were the antimicrobial agents studied. In control mice, the largest drop in lactose fermenting species (1-7-2-8 logs) occurred on day 8 after irradiation and was specifically linked to radiation doses. After day 8, the levels of lactose fermenting organism rose and the increase was correlated with mortality due to Enterobacteriaceae sepsis. Irradiation decreased the populations of strict anaerobic bacteria in control mice by 2-8 logs and stayed low. Treatment with either metronidazole or penicillin resulted in larger decreases in strict anaerobic bacteria than in the controls, leading to earlier and larger changes in lactose fermenting species and subsequent mortality. Treatments with either gentamicin or ofloxacin resulted in smaller declines in strict anaerobic bacteria (11-2-2 logs) than in controls and improved decreases in lactose fermenting species and mortality. Changes in bacterial flora and mortality following imipenem therapy were close to controls. These results suggest that in animals subjected to irradiation, antimicrobial 
agents that are effective against strict anaerobic bacteria may be deleterious, but antimicrobial agents that are effective against lactose fermenting species may be helpful.

The results presented support the correlation between radiation exposure levels and bacterial translocation and Enterobacteriaceae-associated mortality (Brook et al, 1986).These decreases were correlated with decreased amounts of gastrointestinal colonisation with Enterobacteriaceae and diminished translocation of these species (Brook et al , 1990; Hammond, 1954) The effectiveness of these two types of agents is due to their selective action against facultative and aerobic Gram-negative bacilli and their lack of activity against strict anaerobic agents. The treatment of patients with mixed aerobic-anaerobic infections requires the use of antimicrobials that are selective against both groups of pathogens (Bartlett et al, 1978); thus, further work is required to create clinical regimens that can successfully treat irradiated patients who create polymicrobial aerobic-anaerobic infection while minimising the impact on their colonisation. This is a clinical evidence of working of the ofloxacin in the gut microbiota. This helps in presenting the support to the correlation of radiation exposure levels and bacterial translocation. This paper will help in the working of ofloxacin against the gut microbiota and provide evidence against the impact of many other antimicrobial agents like ofloxacin on intestinal microbial flora, systemic infection and mortality in mice subject to elevated doses of radiation.

\section{Synthesis and Antibacterial Activities of Optically Active Ofloxacin}

Two optically active (100\% enantiomeric excess) isomers of ofloxacin $\{(+-)$-ofloxacin; DL-8280; (-+-)9-fluoro-2,3-dihydro-3-methyl-10-(4-methyl-1-

piperazinyl)-7-oxo-7H-pyrido[1,2,3-de]

benzoxazine-6-carboxylic acid\} have been formulated using their optically-resolved synthesised intermediates. One of the isomers, (-)-ofloxacin, was 8 to 128 times more effective in hindering the replication of gram-positive and gramme-negative bacteria than the other, $(+)$-ofloxacin, and roughly 2 times more active than the racemate, $(+)$-ofloxacin. Since nalidixic acid was discovered to have effective action towards gram-negative bacteria, several compounds with 1,4-dihydro-4-oxopyridine-3carboxylic acid ligand, which is important for antibacterial activity, have been synthesised. New 4quinolones with excellent antibacterial activity against not only gram-negative but also gram-positive species have newly been created. The $\mathrm{N}-1$ substituent of 1,4-dihydro-4-oxopyridine-3-carboxylic acid ligand has been stated to be an ethyl group or some other sterically comparable substituent, such as vinyl, fluoroethyl, methoxy, methylamino or cyclopropyl. These replacements do not have an asymmetric middle. On the other hand, ofloxacin 1, a new 4quinolone characterised by a tricyclic form, has a methyl group at the $\mathrm{C}-3$ position in the oxazine ring, leading in an asymmetric centre at that position. Flumequine has a similar asymmetric core as well.

\section{(+) -3,5-Dinitrobenzoyl derivative 3 of racemic} alcohol 2 (I. Hayakawa and Y. Tanaka, Jpn. Kokai Tokkyo Koho JP 591489 [84 1489]) was fully resolved by high-pressure liquid chromatography; solvent, nhexane-1,2-dichloroethane-ethanol (6:3:1); flow rate, $8 \mathrm{ml} / \mathrm{min}$ ) to generate optically pure benzoyl esters $4 \mathrm{a}$ and $4 \mathrm{~b}$. Benzoyl ester 4a was hydrolysed with ethanolic aqueous solution $\mathrm{NaHCO} 3$ at $50^{\circ} \mathrm{C}$ for $2 \mathrm{~h}$ to produce alcohol $5 \mathrm{a}$, which had been modified to iodide $6 \mathrm{a}$ with triphenylphosphine methiodide in $\mathrm{N}$-N-dimethylformamide (DMF) at ambient temperature for $1.5 \mathrm{~h}$. Optically active carboxylic acid 8a was obtained by reducing iodide 6a with tri-n-butyltin hydride in ethanol, followed by hydrolysis of the resulting ester $7 \mathrm{a}$ in concentrated $\mathrm{HCI}-\mathrm{CH} 3 \mathrm{COOH}(2: 1)$ at $120^{\circ} \mathrm{C}$ for $1 \mathrm{~h}$. Compound 
$8 \mathrm{a}$ reacts with 1-methylpiperazine in dimethyl sulfoxide at $120^{\circ} \mathrm{C}$ for $1 \mathrm{~h}$ generated by $(+)$-ofloxacin 9a. Similarly, benzoyl ester $4 \mathrm{~b}$ was modified to (-)ofloxacin $9 b$ by compounds $5 b, 6 b, 7 b$ and $8 b$.

Their interest was directed to the relationship between two optical isomers of ofloxacin and their antibacterial potencies. In this paper, they reported the synthesis of optically active (+)-ofloxacin and (-)ofloxacin through optical resolution of the racemic intermediate of ofloxacin 1 by high-pressure liquid chromatography. This paper tells which of this optical ofloxacin is a potent drug to fight against bacteria. Which one of the optical ofloxacin works on micro gut biota? The results indicate that the configuration of the methyl group at the $\mathrm{C}-3$ position of ofloxacin has significant effects on the antibacterial activity.

\section{Effect of oral ofloxacin on fecal bacteria in human volunteers}

Intestinal members of the Enterobacteriaceae family have been excreted in five human volunteers infected with oral ofloxacin for 5 days. No development of resistant Enterobacteriaceae has been observed. Group D counts of streptococci have been drastically decreased. Colonization of Candida sp. was found in all five volunteers during ofloxacin therapy. The anaerobic flora was reasonably stable from one study to another prior to treatment and was not significantly modified by ofloxacin.

The possible therapeutic advantages of fluoroquinolones involve their use in serious bacterial gastroenteritis and selective decontamination of the gastrointestinal tract of neutropenic patients. Abolishment of faecal aerobic gram-negative bacteria and consequent stabilisation of anaerobic flora after consumption of norfloxacin or ciprofloxacin by human volunteers. It is presumably linked to the enterohepatic circulation of fluoroquinolones.
Because another new fluoroquinolone, ofloxacin, is more active in vitro against anaerobic bacteria than other fluoroquinolones. Five safe, fully aware adults administered oral $200 \mathrm{mg}$ ofloxacin twice daily for 5 days. None of the antimicrobial agents had been taken at least 1 month before the analysis. Blood samples were taken on the first and last days of treatment at $1 \mathrm{~h}$ after the ingestion of the drug. Freshly passing faecal samples were extracted once before surgery, every day, during surgery, and periodically for 1 week afterwards. Fecal anaerobic gloves were counted on Aranki agar in an anaerobic glove box. A total of 814 clones collected from 10-8 or 10-9 dilutions of samples acquired before and after 4 days of treatment ( $82 \pm 7$ clones per sample) were categorised under anaerobic and aerobic conditions according to their Gram staining properties, form and location of the spores and growth characteristics. Anaerobes have been identified as oxygen-sensitive or-tolerant strains, depending on their tolerance to 1 $\mathrm{h}$ of exposure to ambient oxygen. This will help in comparison among fluoroquinolones. And shows that why ofloxacin is the most active in vitro against anaerobic bacteria than others. It's impact on gut microbiota.

\section{Comparative Effects of Levofloxacin and Ofloxacin on the Normal Oral and Intestinal Microflora}

Ever since the introduction of antimicrobial agents in the clinic, antimicrobial resistance has arisen among bacteria. There was no identification of levofloxacin or ofloxacin from saliva samples obtained on days 0,9 , 11, 14 and 21. The ability of an antimicrobial agent to interact with normal microflora is connected to the active concentration at the site of action of the agent. Protection against invasion by potentially pathogenic microorganisms is one of the main functions of normal microflora. The findings of the current study suggest that the administration of levofloxacin and ofloxacin has a selective effect primarily targeted at 
Gram-negative aerobic microflora and does not substantially suppress oral or intestinal anaerobic microflora. The fact that no collection of quinoloneresistant bacteria occurred during the study period reflects this. Ever since the introduction of antimicrobial agents in the clinic, antimicrobial resistance has arisen among bacteria. As a result of the widespread use of antimicrobial agents, it seems that resistance is emerging at a faster pace than ever before. Antimicrobial resistance can cause severe infections and treatment failures in clinical isolates from the site of infection and can lead to the use of higher doses or more toxic alternative drugs. The choice of a therapeutic agent, based on the expected clinical response and the degree of expected ecological disruptions, such as the production of resistance, is very important to consider carefully. This paper provides clinical evidence on of having selective effect primarily targeted at Gram-negative aerobic microflora and not having substantially suppressed oral or intestinal anaerobic microflora. It will help me in comparison with cephalosporin as one of its group is effective against the gram-negative bacteria.

\section{Cefixime}

\section{Cefixime in the Treatment of Respiratory and Urinary Tract Infections}

The pilot study reveals the wide usage of cefixime for various infections and because of lower adverse effect cefixime was recommended to use at any age levels. They conducted this study in 4 European countries, because of the sudden cefixime establishment in western area. A total of 252 patients with RTI and UTI evaluated for the study. 95 children were enrolled with upper RTI, whereas 60 adults with AECB record, 12 with pneumonia and 85 with UTI were volunteered. Patients was administered standard cefixime of $400 \mathrm{mg}$ once in a day orally and $8 \mathrm{mg} / \mathrm{kg}$ body weight in suspension for children. The treatment duration for UTI and RTI was 3-7 days and 5-10 days respectively. Bacteriological testing was carried out after the treatment period. They exhibited a tremendous improvement in $90 \%$ of the patient treated, only few resulted failures. The microbial results in RTI showed eradiation of 40/45, 35/36 from children and 64/71 from UTI patients. These reports support the usage of cefixime for various treatments. The clinical study of antibiotics in a wide place provides a support to use these drugs for various infections and this is reported to be a pilot study.

\section{Mechanism of Therapeutic Effectiveness of Cefixime against Typhoid Fever}

This article opens a way to understand the therapeutic effects of cefixime for the treatment of typhoid fever. Clinical efficacy of cefixime against typhoid fever was confirmed in vivo study using mouse systemic infection model (Male 4-week-old ICR mice) caused by several strains of serovar Typhimurium FP39, FP1671, and LT2. Upon 3 days of cefixime treatment, observed a potent therapeutic activity with a $50 \%$ effective dose (ED50) of 1.48 $\mathrm{mg} / \mathrm{kg}$ at 14 days after infection. In vitro THP-1 cells viability was observed after 6hrs serovar Typhimurium FP39 infection and measured using LIVE/DEAD cytotoxicity kit and analysis was done in FACS. They carried a microscopic study to find the apparent morphological changes that occurs after cefixime induction into the THP-1 cells. They observed an elongation in serovar Typhimurium FP39 inside THP-1 cells though $48 \%$ of cefixime concentration was only observed inside the cells but it was sufficient to create real damage to serovar Typhimurium. A fair amount of cefixime can inhibit the bacterial growth inside the cells supports the therapeutic effectiveness of cefixime over typhoid fever. 
They have proved the clinical application of the drug in vivo and in vitro. The techniques used for the study are essential to find the molecular action of a drug. Most interesting was the usage of FACS. This technique helps in sorting the cells from a given population and even it can be used for the stem cell isolation which is an upcoming therapeutic target for cancer therapies.

Comparative in vitro antibacterial analysis of different brands of cefixime against clinical isolates of Staphylococcus aureus and Escherichia coli

The over usage of antibiotics develops resistance to drugs thereby promoting the bacterial growth and causes health problems. The comparison of different brand of cefixime against clinical and standard isolates of microorganism was evaluated using Muller Hinton method and UV-spectrophotometer. These standard and clinical isolates were cultured in medium and each concentration of standard cefixime and different brands was added respectively to the inoculum and incubated at $37^{\circ} \mathrm{C}$ for 18 hours and reading was noted. As per previous report by Neu et al., same range of minimum inhibitory concentration of standard cefixime for E.coli was observed in the same study. Standard cefixime showed MIC ranging from $8-64 \mu \mathrm{g} / \mathrm{ml}$ for five E-coli clinical isolates and 8 $-128 \mu \mathrm{g} / \mathrm{ml}$ for $\mathrm{S}$. aureus clinical isolates. The MIC of different cefixime brands for the E-coli clinical isolates and $\mathrm{S}$. aureus ranges 8-32 $\mu \mathrm{g} / \mathrm{ml}$ and $8-256$ $\mu \mathrm{g} / \mathrm{ml}$ respectively. The higher MIC values indicating that both organisms developed resistance to cefixime.

These techniques help to do the comparison study of different antibiotics against various organisms and the concentration can be assessed using spectrophotometer. Various studies are undergoing on drug delivery system and this will favour to understand activity of the drug on specific organism.
Efficacy of cefixime in the treatment of Urinary Tract Infection

Urinary tract infections (UTI) are most common bacterial infections affecting people worldwide, so considered to be a severe public issue. While the incidence of UTI in men aged under 50 is low, adult women is $30 \%$ more likely than men to develop UTI, moreover elderly patients are more susceptible. Cefixime shown to have a broad spectrum of antibacterial activity against both gram positive and gram-negative bacteria, therefore it is considered as a potent antibiotic for the treatment of UTIs. The aim of this study was to determine the efficacy of cefixime in UTI patient is enrolled from different hospitals of Nepal. 65 UTI patients were selected randomly, 41 were females and 24 patients were males. The patients were diagnosed with UTI by urine culture analysis. They categorised the patients according to the age, gender and occupation (student, housewife, employee, and farmer). Later, patients received cefixime was observed for 10 days of period and suggested follow-up on $3^{\text {rd }}, 5^{\text {th }}$ and $7^{\text {th }}$ day of treatment. The statistical interpretation was carried using SPSS. The result suggests that cefixime administration cured completely among 61 of 65 patients and showing efficacy of 93.85\%. Though treatment was effective, it showed some side effects like nausea, gastritis, rashes and drowsiness during treatment but these were reported to be in minor amount. This study not only evaluates the efficacy of cefixime against UTI but also showed the tolerance and safety level in patients. Therefore, cefixime proved to be a good alternative for the treatment of UTI.This is another study confirms the potential activity of cefixime against microbial infections. They conducted this study in different hospitals of Nepal, 65 patients of both genders were enrolled for the study. The categorised UTI patients were administered with $200 \mathrm{mg}$ of cefixime every $12 \mathrm{hrs}$ for 7-10 days. The patients were subjected to assessment on $7^{\text {th }}$ and $10^{\text {th }}$ day during treatment. Highest number 
of patients were found to be female house makers. Out of 65 patients, few showed side effects, nausea (1 patient), gastritis (2 patient), rashes (2 patients) and drowsiness (1 patient). No other side effects were reported in remaining 59 patients. Interestingly, 61 patients of 65 showed a complete recovery, 1 does not show any therapeutic changes and 3 did not followed up after treatment. Thus, efficacy of cefixime was higher showed to be an effective drug for the UTI treatment.

Microbial urine culture was majorly used for the diagnosis of bacterial infections. This is very essential to detect the specific species causing infections.

\section{Synthesis of Cefixime and Azithromycin} Nanoparticles: An Attempt to Enhance Their Antimicrobial Activity and Dissolution Rate

Several drugs were shown to have low water solubility and low dissolution rates. Cefixime is considered as a low solubility and low permeability cephalosporin antibacterial drug. Due to low solubility of cefixime, its bioavailability is only $30-50 \%$ of an oral dose absorbed and shows maximum peak serum concentrations within 2-6 hours. Azithromycin is also a poorly water-soluble, semisynthetic macrolide antibiotics. It is absorbed rapidly after oral administration with a bioavailability of about $36 \%$. The solubility and dissolution rate of these drugs can be enhancing by reducing particle size. The nanoparticles have different physicochemical and physiological properties, such as reduced light scattering, improved stability to gravitational separation and aggregation, faster diffusion rates, higher solubility, and higher penetration rates through biological barriers compared to the larger particles. In this study they prepared cefixime and azithromycin nanoparticles by antisolvent precipitation with syringe pump (APSP) and evaporator precipitation nanosuspension (EPN) methods. The developed nanoparticles were characterized by XRD, FTIR, SEM, and TGA. Cefixime showed the amorphous form whereas, azithromycin samples showed crystalline form in the $\mathrm{X}$-ray diffraction pattern. No major structural changes were detected in the FTIR spectra of parental drugs and synthesized nanoparticles. The SEM analysis were carried out to determine the morphology of nanoparticles of cefixime, the images of nanoparticles of both drugs showed a submicron sized and nanosized particles. The results of TGA analysis showed that cefixime sample weight decreases gradually when the temperature increases up to $600{ }^{\circ} \mathrm{C}$, while, in case of azithromycin from above $250{ }^{\circ} \mathrm{C}$ decomposition of the sample took place. Agar well diffusion method was used to determine the antibacterial activity of raw drugs and prepared nanoparticles sample against Staphylococcus aureus, Shigella, E. coli, and Salmonella typhi. The synthesized nanoparticles showed good dissolution rate than raw drugs. Therefore, this study revealed that reduced size of the parental drug resulted in enhanced antimicrobial activity and dissolution rate. Also, antimicrobial activities of nanoparticle sample of cefixime and raw drug against bacterial strains, all the results showed a higher activity of prepared nanoparticle samples than raw drug. Same trend was observed in dissolution study, where nanoparticles has higher dissolution rate than raw drug. Altogether, synthesized nanoparticles always showed a better result than parental drug. Since nanoparticles are more effective for drug delivery, these methods are widely used in the pharmaceutical industry in order to understand each and every characteristics of synthesized drug.

\section{Increase in Bacteroides/firmicutes ratio after early- life repeated administration of Cefixime in Rat.}

The usage of antibiotics increases day by day with the severity of health issues. Antibiotics has beneficial effects, but long-term usage can shift its role and 
causes severe alterations in normal gut microbiota. Also, reports states that early usage of antibiotics in children could affect the metabolic development and causes significant health implications, such as obesity and Type II Diabetes later in life. Cefixime is an antibiotic widely used to treat respiratory tract and gastrointestinal infections, common diseases found in children. Unfortunately, there are no reports available on the effect of cefixime to gut microbial diversity. So, they evaluated the changes in gut microbial diversity after continuous exposure of cefixime in early life. To do the study, they have used six-week-old male wistar rats as experimental model and randomly divided the rats into control group and cefixime group. Prior to exposure of antibiotic, the rats were transplanted with faeces orally from 7months old boy. Cefixime was given 3 consecutive periods with one-week interval. Rats were sacrificed after the treatment and caecal content was collected to determine the changes in gut microbiota composition using Microbiome analysis. They found an increase in relative abundance of Bacteroidetes and a decrease in relative abundance of firmicutes upon repeated administration of cefixime. They also observed an increase in the ratio by $32 \%$ of Bacteroidetes/Firmicutes. These findings are consistent with the previous reports using $\beta$-lactam antibiotics as exposing agents. However, they concluded that early antibiotic administration alters gut microbial composition in vivo but further research is warranted to understand the metabolic changes in gut microbiota. Techniques like, Next generation sequencing (NGS) is an emerging tool for the investigation of genome diversity and to find the epigenetic alterations and gene expression profiling by RNA. Various mutations in genome causes several health abnormalities. This technique helps to find cancer related mutations and early detection of aging in cells.

\section{Conclusion:}

The Review paper shows us the strong relationship of these antibiotics and their effect on gut microbial flora, discussing about both positive and adverse effects.

\section{Acknowledgement:}

We would like to thank our supervisor, Professor Bharat Kwatra, from Invenzion Labs. whose expertise was invaluable in formulating the research questions, methodology and drawing Conclusions. His insightful feedback and Guidance pushed me to sharpen our thinking and brought our work to a higher level.

\section{REFERENCES}

[1]. Edlund, C. et al. Comparative Effects of Moxifloxacin and Clarithromycin on the Normal Intestinal Microflora. Scand J Infect Dis vol. 32 (2000).

[2]. Edlund', C., Sjostedt2, S., Nord', C. E. \& Nord, C. E. Comparative Effects of Levofloxacin and Ofloxacin on the Normal Oral and Intestinal M i crof I o ra. Scand J Infect Dis vol. 29 (1997).

[3]. Iizumi, T. et al. Effect of antibiotic pretreatment and pathogen challenge on the intestinal microbiota in mice. Gut Pathog. 8, 110 (2016).

[4]. Hooton, T. M., Roberts, P. L. \& Stapleton, A. E. Cefpodoxime vs Ciprofloxacin for Short-Course Treatment of Acute Uncomplicated Cystitis A Randomized Trial. 5JAC445647.

[5]. Pecquet, S., Andremont, A. \& Tancrede, C. Effect of oral ofloxacin on fecal bacteria in human volunteers. Antimicrob. Agents Chemother. 31, 124-125 (1987).

[6]. Hayakawa, I., Atarashi, S. \& Yokohama, S. Synthesis and Antibacterial Activities of 
Optically Active Ofloxacin. ANTIMICROBIAL AGENTS AND CHEMOTHERAPY (1986).

[7]. Hardy, D., Amsterdam, D., Mandell, L. A. \& Rotstein, C. and Other Antimicrobial Agents against Bloodstream Isolates of Gram-Positive Cocci. vol. 44 (2000).

[8]. Brook, I. \& David Ledney, G. Effect of antimicrobial therapy on the gastrointestinal bacterial flora, infection and mortality in mice exposed to different doses of irradiation. Journal of Antimicrobial Chemotherapy vol. 33 (1994).

[9]. Lang, R., Lbhner, M., Segev, S. \& Rubinstein, E. Ofloxacin and the gastrointestinal tract: a potential role in the treatment of bacterial enteritis. Journal of Antimicrobial Chemotherapy vol. 26 (1990).

[10]. Chachaty, E. et al. Presence of Clostridium di;icile and Antibiotic and ,B-Lactamase Activities in Feces of Volunteers Treated with Oral Cefixime, Oral Cefpodoxime Proxetil, or Placebo. ANTIMICROBIAL AGENTS AND CHEMOTHERAPY vol. 36 (1992).

[11]. Brismar, B., Edlund, C. \& Nord, C. E. Impact of Cefpodoxime Proxetil and Amoxiciilin on the Normal Oral and Intestinal Microflora. New Antimicrobial Agent vol. 12.13603 (2).

[12]. Hughes, G. S. et al. The effects of gastric $\mathrm{pH}$ and food on the pharmacokinetics of a new oral cephalosporin, cefpodoxime proxetil The effects of alteration of gastric $\mathrm{pH}$ and food on the pharmacokinetics of $200 \mathrm{mg}$ doses of cefpodoxime.

[13]. Ghosh, A. \& Chatterjee, S. Comparison of efficacy and safety of cefpodoxime and amoxicillin-clavulanate potassium in paediatric acute otitis media in children below two years: A prospective longitudinal study. J. Clin. Diagnostic Res. 11, FC01-FC04 (2017).

[14]. Wiedemann, B. \& Jansen, A. Antibacterial activity of cefpodoxime proxetil in a pharmacokinetic in-vitro model. Journal of Antimicrobial Chemotherapy vol. 26 (1990).

[15]. Cox, C. E., Graveline, J. F. \& Luongo, J. M. Review of Clinical Experience in the United States with Cefpodoxime Proxetil in Adults with Uncomplicated Urinary Tract Infections. Drugs vol. 42 (1991).

[16]. Cefpodoxime: Comparative Antibacterial Activity, Influence of Growth Conditions, and Bactericidal Activity.

[17]. Stentz, R. et al. Cephalosporinases associated with outer membrane vesicles released by Bacteroides spp. protect gut pathogens and commensals against $\beta$-lactam antibiotics. J. Antimicrob. Chemother. 70, 701-709 (2015).

[18]. Chugh, K. \& Agrawal, S. Cefpodoxime: Pharmacokinetics and Therapeutic Uses.

[19]. Khan, F. A., Zahoor, M., Islam, N. U. \& Hameed, R. Synthesis of Cefixime and Azithromycin Nanoparticles: An Attempt to Enhance Their Antimicrobial Activity and Dissolution Rate. J. Nanomater. 2016, (2016).

[20]. Pinatih, K. J. P., Suastika, K., Sukrama, I. D. M. \& Sujaya, I. N. Increase in bacteroides/firmicutes ratio after early-life repeated administration of Cefixime in Rat. Bali Med. J. 6, (2017).

[21]. Chaudhary, M. et al. Antihypertensive medication prescribing patterns in crimson hospital View project EFFICACY OF CEFIXIME IN THE TREATMENT OF URINARY TRACT INFECTION. www.wjpps.com vol. 4 (2015).

[22]. Matsumoto, Y. et al. Mechanism of therapeutic effectiveness of cefixime against typhoid fever. Antimicrob. Agents Chemother. 45, 2450-2454 (2001).

[23]. Krishnan, P., Stalin, M. \& Balasubramanian, S. Changing trends in antimicrobial resistance of Salmonella enterica serovar typhi and salmonella enterica serovar paratyphi $\mathrm{A}$ in 
Chennai. Indian J. Pathol. Microbiol. 52, 505508 (2009).

[24]. Arshad, H. M., Mohiuddin, O. A. \& Azmi, M. B. Comparative in vitro antibacterial analysis of different brands of cefixime against clinical isolates of Staphylococcus aureus and Escherichia coli. J. Appl. Pharm. Sci. 2012, 109113.

[25]. Ludwig, E. \& Ludwig, E. Cefixime in the Treatment of Respiratory and Urinary Tract Infections. Chemotherapy vol. 44 (1998).

[26]. Knapp, C. C., Sierra-Madero, J. \& Washington, J. A. Antibacterial Activities of Cefpodoxime, Cefixime, and Ceftriaxone. ANTIMICROBIAL AGENTS AND CHEMOTHERAPY vol. 32 (1988).

[27]. Counts, G. W., Baugher, L. K., Ulness, B. K. \& Hamilton, D. J. New Antimicrobial Agents Comparative in Vitro Activity of the New Oral Cephalosporin Cefixime. J. Clin. Microbiol. Infect. Dis.

[28]. Moazzezy, N., Karam, M. R. A., Rafati, S., Bouzari, S. \& Oloomi, M. Inhibition and eradication activity of truncated $\alpha$-defensin analogs against multidrug resistant uropathogenic Escherichia coli biofilm. PLoS One 15, (2020).

[29]. Kwatra, B. A Review on Potential Properties and Therapeutic Applications of DHA and EPA. ijppr.humanjournals 16, 140-176 (2019).

[30]. Kwatra, B. Tinospora Crispa As A Future Cure For Obesity/Cholesterol. Int. J. Sci. Technol. Res. 6, 340-341 (2017).

[31]. Kwatra, B. A REVIEW ON POTENTIAL PROPERTIES AND THERAPEUTIC APPLICATIONS OF BROMELAIN. www.wjpps.com 8, 488-500 (2019).

[32]. Kwatra, B. Holothuroidea (Sea Cucumber): Key to Anti-Aging. Int. J. Sci. Res. 8, 884-884 (2019).
[33]. Kwatra, B. \& Mudgil, M. PROTONATED CRAB SHELL WASTE AS FUNGAL INHIBITOR. Int. J. Med. Biomed. Stud. 3, 111-116 (2019).

[34]. Kwatra, B. A REVIEW ON POTENTIAL PROPERTIES AND THERAPEUTIC APPLICATIONS OF GRAPE SEED EXTRACT. World J. Pharm. Res. 9, 2519-2540 (2020).

[35]. Kwatra, B. A REVIEW ON POTENTIAL PROPERTIES AND THERAPEUTIC APPLICATIONS OF BRANCHED CHAIN AMINO. WORLD J. Pharm. Pharm. Sci. 9, 561588 (2020).

[36]. Kwatra, B. COLLAGEN SUPPLEMENTATION : THERAPY FOR SKIN DISORDERS: A REVIEW. World J. Pharm. Res. 9, 2504-2518 (2020).

[37]. Kwatra, B. A REVIEW ON POTENTIAL PROPERTIES AND THERAPEUTIC APPLICATIONS OF LYCOPENE. Int. J. Med. Biomed. Stud. 4, 33-44 (2020).

[38]. Kwatra, B. \& Mudgil, M. LIGHT ASSISTED TIO2-BASED NANOCOMPOSITE SYSTEMS: A NOVEL TREATMENT FOR CANCER. Int. J. Med. Biomed. Stud. 4, 28-32 (2020).

[39]. Kwatra, B. A Review on Potential Properties and Therapeutic Applications of Vitamin D. Int. J. Sci. Res. 9, 682-691 (2020).

[40]. Kwatra, B. HYDROQUINONE: A novel growth inhibitor and apoptosis inducer in U-251 MG CELLS. Int. J. Med. Biomed. Stud. 3, 15-16 (2019).

[41]. Bharat Kwatra. Procuring Natural Dye for Solar Cell Using Leaf Waste. Int. J. Sci. Eng. Res. 7, 46-47 (2019).

[42]. Kwatra, B. HACKING THE BLOOD-BRAIN BARRIER. Eur. J. Biol. Med. Sci. Res. 5, 10-13 (2017).

[43]. Kwatra, B. EXPRESSION AND CHARACTERIZATION IN PICHIA PASTORIS BY CLONING OF DELTA 4 DESATURASE 
FROM ISOCHRYSIS GALBANA. Indian J. Appl. Res. 9, 1-2 (2019).

[44]. Kwatra, B. CALCIUM AND IRON ABSORPTION: INVITRO STUDIES. Int. J. Med. Biomed. Stud. 3, 59-61 (2019).

[45]. Kwatra, B. Allicin-An After Digestion Antimicrobial Agent. ACTA Sci. Microbiol. 2, 48-51 (2019).

[46]. Kwatra, B. LOCATOR THEORY FOR ELEMENTS IN PERIODIC TABLE 'LEPT'. Glob. J. Pure Appl. Chem. Res. 5, 9-10 (2017).

[47]. Kwatra, B. \& A, A. UTERINE CANCER: SEX DOMINANT CHARACTER. Int. J. Adv. Res. 08, 663-667 (2020).

[48]. Kwatra, B. Studies on People Employed in High Risk Workplace: Between Genetic Polymorphism for Tumor Necrosis Factor ( TNF- A ) and Blood Pressure. Int. J. Innov. Res. Technol. 6, 268-270 (2020).

[49]. Kwatra, B. \& Arora, C. Investigation of Conductance Quantization with Magnetic Field Control and Application of Biosensor. Int. J. Innov. Res. Technol. 6, 271-272 (2020).

[50]. Kwatra, B. Candidate genes of OCD interacts with human retrovirus to form new link in inflammatory hypothesis. Int. J. Sci. Appl. Res. 7, 1-2 (2020).

[51]. Kwatra, B. COLLAGEN SUPPLEMENTATION : THERAPY FOR THE PREVENTION AND TREATMENT OF OSTEOPOROSIS AND OSTEOARTHRITIS: A REVIEW. WORLD J. Pharm. Pharm. Sci. 9, 589-604 (2020).

[52]. Kwatra, B. THE SIMVASTATIN AND DMXAA ON THE CO-CULTURE OF B16.F10 MELANOMA CELLS AND MACROPHAGES SHOWS ANTITUMOR ACTIVITY. World J. Pharm. Res. 8, 1318-1319 (2019).

[53]. Kwatra, B. Bioactive-Compounds: alternative to control Candida spp. Int. J. Sci. Res. Rev. 8, 221-223 (2019).
[54]. Kwatra, B. Effects of Mineral Separation by Time and Enteric Coating Mechanism for Calcium and Iron Absorption in Mammalia. Int. J. Sci. Res. 8, 1265-1270 (2019).

[55]. Kwatra, B. Maprovit 3, 6, 9: Perfect Companion of your Immune System to Fight Corona Virus Hit. Int. J. Sci. Res. 9, 241-241 (2020).

[56]. Kwatra, B. \& Mudgil, M. Untangling the Mathematical Relation Between Natural Selection and Adaptive Radiation Using Macromolecules and Microevolutionary Forces. Int. J. Sci. Res. Sci. Technol. IJSRST | 7, 313-339 (2020).

[57]. Kwatra, B. MECHANISMS OF PATTERN FORMATION OF FBP17 IN MAST CELLS. Int. J. Adv. Res. 7, 413-414 (2019).

[58]. Mudgil1, M. \& Kwatra2, B. Mosquito Menace Aim: Observing the life cycle of Aedes aegypti mosquito and understanding its behavior towards different natural oils for encouraging natural methods of repellence. Int. J. Sci. Res. 8, 1314-1315 (2019). 1,2,11-20,3,21-30,4-1031-60

\section{Cite this article as :}

Kartik Singh, Manju Mohan, Shrishti Nautiyal, "Comparing Cefixime, Cefpodoxime and Ofloxacin as Anti-Microbial Agents and their Effects on Gut Microbiota", International Journal of Scientific Research in Science and Technology (IJSRST), Online ISSN : 2395-602X, Print ISSN : 2395-6011, Volume 7 Issue 6, pp. 131-152, November-December 2020. Available at doi : https://doi.org/10.32628/IJSRST207619 Journal URL : http://ijsrst.com/IJSRST207619 